\title{
Unexpected deaths following discharge of medical patients from hospital
}

\section{Introduction}

Unexpected death of a patient shortly following discharge from hospital is one of the worst nightmares for a clinician who had been involved in the discharge decision. Indeed, any adverse event post discharge could be stressful for the patients and the clinical team. ${ }^{1}$ Though there is data on hospital readmission rates and post-discharge adverse events, we do not have data on the incidence of unexpected deaths following discharge of medical inpatients. ${ }^{2-4}$ Hence, we undertook a prospective observational study to estimate the incidence of deaths within 72 hours of discharge from the acute hospital.

\section{Methods}

The study was performed at a district general hospital serving a population of over 220,000 . We sought permission from the hospital's medical directorate and the senior coroner for North Wales. This study complemented the hospital's routine mortality reviews. We identified patients who died within 72 hours of discharge through the hospital's bereavement office and the coroner's office.

We only included deaths following discharge from the medical teams. We excluded deaths following emergency department attendance, anticipated deaths (end-of-life care), deaths in community hospitals following transfer from the acute site and deaths following non-medical specialty discharges.

\section{Results}

Over 16 months (March 2018 - June 2019), nine unexpected deaths occurred within 72 hours of discharge from the medical teams. During that period, there were around 13,000 medical discharges, giving an incidence of $0.06-0.07 \%$ or around one death per 1,300 discharges.

The mean age of the patients was 68 years (range $34-88$ years; eight males and one female). Eight patients were discharged by consultants and one by a middle grade trainee. The average duration between discharges to death was 1.2 days (a few hours to 2 days). The mean pre-discharge National Early Warning Score (NEWS) was $1.7(0-5)$. The average length of stay before discharge was 7 (0-15) days.

All cases were discussed with the coroner, and five patients underwent post-mortem examination. For the other four patients, clinicians were able to provide a death certificate. Only one discharge was deemed to be unsafe. Except for one patient, the rest had multiple comorbidities and the death didn't come as a surprise.

The primary causes of death.

$>$ Ruptured aortic aneurysm.

$>$ Metastatic cancer (known diagnosis).
> Myocardial infarction/coronary artery disease (two cases).

$>$ Cardiac arrhythmia.

$>$ Cardiac failure.

$>$ Bronchopneumonia.

$>$ Pulmonary embolism.

$>$ Drug overdose (known abuser).

\section{Discussion}

From our study, the risk of unexpected death following discharge from acute hospital medical wards is around 1 in 1,300. Since there is no published data, we were unable to compare our figures with other regions and countries. All deceased patients were discharged by a senior doctor and the NEWS was low in many of them.

While it is reassuring that unexpected mortality following discharge of medical inpatients is low, it does happen regularly and bound to very stressful. This data would be very useful when discussing such risks with patients, families, coroners, litigation and legal services and for the wider governance structure. Clinicians should keep clear and accurate records when discharging patients as these will be crucial to defend and reflect on decisions and actions when such unexpected adverse incidents occur.

Vedamurthy Adhiyaman Consultant geriatrician, Glan Clwyd Hospital, Rhyl, UK Indrajit Chattopadhyay Consultant geriatrician, Glan Clwyd Hospital, Rhyl, UK

\section{References}

1 Oliver D. The stress of sending patients home. BMJ 2019;365:I2094.

2 NHS Digital. Emergency readmissions published for first time in five years. NHS, 2019. https://digital.nhs.uk/news-and-events/latestnews/emergency-readmissions-published-for-first-time-in-five-years.

3 Forster AJ, Murff HJ, Peterson JF, Gandhi TJ, Bates DW. The incidence and severity of adverse events affecting patients after discharge from the hospital. Ann Intern Med 2003;138:161-7.

4 National Institute for Health and Care Excellence. Chapter 35 Discharge planning: Emergency and acute medical care in over 16s: service delivery and organisation: NICE guideline 94. NICE, 2017. https://www.nice.org.uk/guidance/ng94/ evidence/35discharge-planning-pdf-172397464674.

Address for correspondence: Dr Vedamurthy Adhiyaman, Department of care of the elderly, Glan Clwyd Hospital, Rhyl LL18 5UJ, UK.

Email: vedamurthy.adhiyaman@wales.nhs.uk 